\title{
Calculation Algorithm of Tire-Road Friction Coefficient Based on Limited-Memory Adaptive Extended Kalman Filter
}

\author{
Bin Huang, ${ }^{1}$ Xiang Fu $\mathbb{D}^{1},{ }^{1}$ Sen $W{ }^{2},{ }^{2}$ and Song Huang ${ }^{2}$ \\ ${ }^{1}$ Hubei Key Laboratory of Advanced Technology for Automotive Components, Wuhan University of Technology, Wuhan, China \\ ${ }^{2}$ Hubei Collaborative Innovation Center for Automotive Components Technology, Wuhan, China \\ Correspondence should be addressed to Xiang Fu; fuxiang@whut.edu.cn
}

Received 1 February 2019; Revised 11 April 2019; Accepted 2 May 2019; Published 14 May 2019

Academic Editor: Alessandro Palmeri

Copyright (c) 2019 Bin Huang et al. This is an open access article distributed under the Creative Commons Attribution License, which permits unrestricted use, distribution, and reproduction in any medium, provided the original work is properly cited.

\begin{abstract}
In this paper, a limited-memory adaptive extended Kalman Filter (LM-AEKF) to estimate tire-road friction coefficient is proposed. By combining extended Kalman filter (EKF) with the limited-memory filter, this algorithm can reduce the effects of old measurement data on filtering and improve the estimation accuracy. Self-adaptive regulatory factors were introduced to weigh covariance matrix of evaluated error. Meanwhile, measured noise covariance matrix was adjusted dynamically by fuzzy inference to accurately track the breaking status of system. Therefore, problems, including large filter error and divergence caused by incorrect model, can be solved. Joint simulation was conducted for the proposed algorithm with Carsim and Matlab/Simulink. Under the different road conditions, real-vehicle road tests were conducted in various working conditions for contrast with traditional EKF results. Simulation and real-vehicle road tests show that this algorithm can enhance the filter stability, improve the estimation accuracy of algorithm, and increase algorithm robustness.
\end{abstract}

\section{Introduction}

With continuous development and application of information technology in automotive field, people have higher requirement for the active safety of automobile. Four-wheel independent driven electric vehicle can realize active safety control by independent control over four-wheel driven torque. It is necessary to obtain the current tire-road friction coefficient to make control system rationally distribute motor torque and fully utilize the friction limit of tire. Therefore, wheel slip or lock can be avoided to ensure the active safety control of the whole vehicle [1-3]. The use of instruments to directly measure pavement information can achieve relatively accurate results, but such sensors have very high requirements on the working environment and are expensive, which limits their application. Therefore, using low-cost on-board sensors to measure vehicle state parameters, and then estimating the state of vehicle dynamics based on the vehicle dynamics equation, and then real-time estimation of pavement information has become a research focus $[4,5]$. Domestic and foreign scholars have made a large amount of studies on tire-road friction coefficient estimation based on $\mu$-s curve [6-11]. However, a large amount of data is required for data fitting because this method has problems of slow response time, weak timeliness, and heavy dependence of identification correctness on model accuracy. As a strong nonlinear complex system, vehicle may cause an inevitable error between the vehicle dynamics model and the real system. Such error restrains the model reflecting the real physical process and fails the matching between observation value and the model. Therefore, the problems, such as large filter error and even divergence, may be caused $[12,13]$.

Based on the vehicle sensor information fusion technology, this paper designs the road surface adhesion coefficient estimator based on the nonlinear vehicle dynamics model and uses the simplified Dugoff tire model to extract the wheel adhesion coefficient variables, using the extended Kalman filter (EKF) theory. By combining EKF with the limitedmemory filter to reduce the effects of old measurement 
data on filtering and improve the estimation accuracy, selfadaptive regulatory factors were introduced to weigh covariance matrix of evaluated error for solving the problems of excessive or even fading due to inaccurate model.

Traditional EKF has high estimation accuracy, but it is unlimitedly growing memory filter as standard Kalman filter (KF). When estimating filter at any time, all the data before this moment should be used, causing the insufficient use of current sensor measurement. When there is model error and unknown time-varying noise in the system, new observation data only plays a small role in correcting state estimation. Therefore, error may accumulate to cause large filter error and even divergence [14-17]. Based on it, this work introduced limited-memory filter based on traditional EKF to increase the role of new observation data and reduce the harmful effects of old measurement data on filter. The performance and accuracy of estimation algorithm can be improved, and the stability of filter can be enhanced. Meanwhile, with fuzzy logic inference, the noise measurement matrix was adjusted online in real time according to the difference between actual variance and theoretical variance of real-time information to improve the self-adaption of algorithm.

In this paper, we proposed a limited-memory adaptive extended Kalman Filter (LM-AEKF) to estimate tire-road friction coefficient. By combining EKF with the limited memory filter, this algorithm can reduce the effects of old measurement data on filtering and improve the estimation accuracy. Self-adaptive regulatory factors were introduced to weigh covariance matrix of evaluated error. Meanwhile, measured noise covariance matrix was adjusted dynamically by fuzzy inference to accurately track the breaking status of system. Therefore, problems including large filter error and divergence caused by incorrect model can be solved. In summary, our contributions are as follows:

(1) An algorithm for enhancing estimation accuracy and robustness is designed.

(2) The paper establishes a 3-DOF nonlinear vehicle dynamics model to better reflect the response characteristics of real vehicles.

(3) By combining EKF with the limited-memory filter, this algorithm can reduce the effects of old measurement data on filtering and improve the estimation accuracy. Selfadaptive regulatory factors were introduced to weigh covariance matrix of evaluated error.

(4) The algorithm has good estimation accuracy and stability, the response speed of the algorithm is improved, and the adaptability is enhanced.

In recent years, study was widely conducted on realtime estimation of road-vehicle friction coefficient based on the information fusion technology of vehicle sensor. A simultaneous online estimation of the vehicle's state and maximum friction coefficient is presented using a joint Unscented Kalman Filter in [18]. Online estimation of the bounded maximum friction coefficient based on serial sensors using a sensitivity-based joint unscented Kalman filter is presented in [19]. The coefficient was initially simulated and estimated based traceless Kalman filter algorithm in [20]. Interactive multimodel algorithm was used to estimate the speed and tire-road friction coefficient in [21]. Tire-road friction coefficient was identified with the least square method of regression and auxiliary variable method in [22]. [23, 24] use the dual unscented Kalman filter theory to estimate the vehicle state and the road surface adhesion coefficient, so that the two-estimators' information is exchanged and corrected, and finally the identification of the current road surface adhesion coefficient is realized.

In summary, scholars have carried out multimethod research on the road surface adhesion coefficient estimation algorithm and achieved certain results. However, the convergence speed and accuracy of these algorithms need to be improved, and the robustness needs to be enhanced.

\section{Nonlinear Vehicle Dynamic Model}

This section analyzes the 3-DOF vehicle dynamics model and the wheel rotation dynamics model, revealing the coupling relationship between the vehicle operating state parameters and the tire-road adhesion coefficient. The purpose is to provide theoretical basis and technical support for the design of the parameter estimator.

2.1. Vehicle Model. This work mainly focuses on the tireroad friction coefficient estimation of vehicle in horizontal direction. The proposed estimation algorithm is based on three-degree freedom vehicle model in vertical (see Figure 1), lateral, and horizontal directions.

The equations in these three movement directions are as follows:

$$
\begin{aligned}
a_{x}= & \frac{1}{m}\left[\left(F_{x 1}+F_{x 2}\right) \cos \delta+F_{x 3}+F_{x 4}\right. \\
& \left.-\left(F_{y 1}+F_{y 2}\right) \sin \delta-F_{w}\right] \\
F_{w} & =\frac{1}{2} C_{D} \rho A v_{x}^{2} \\
a_{y}= & \frac{1}{m}\left[\left(F_{x 1}+F_{x 2}\right) \sin \delta+\left(F_{y 1}+F_{y 2}\right) \cos \delta+F_{y 3}\right. \\
& \left.+F_{y 4}\right] \\
r= & \frac{1}{I_{z}}\left\{a\left[\left(F_{x 1}+F_{x 2}\right) \sin \delta+\left(F_{y 1}+F_{y 2}\right) \cos \delta\right]\right. \\
& -b\left(F_{y 3}+F_{y 4}\right) \\
& -\frac{t_{f}}{2}\left[\left(F_{x 1}-F_{x 2}\right) \cos \delta-\left(F_{y 1}-F_{y 2}\right) \sin \delta\right] \\
& \left.-\frac{t_{r}}{2}\left(F_{y 3}-F_{y 4}\right)\right\}
\end{aligned}
$$

where $a_{x}, a_{y}$, and $r$ refer to the vertical accelerated velocity, lateral accelerated velocity, and horizontal angular velocity, respectively; $\mathrm{m}$ is the vehicle total mass; $F_{x i}$ and $F_{y i}$ are 


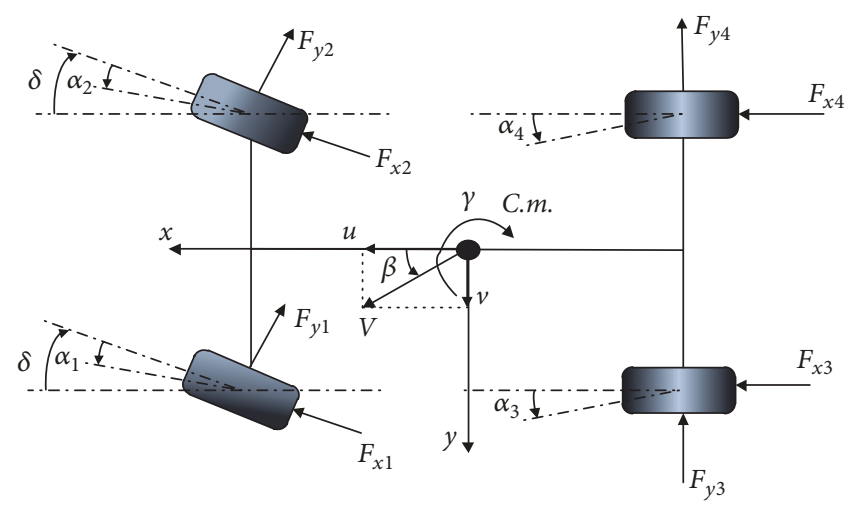

FIGURE 1: Three-degree freedom vehicle model.

longitudinal force and lateral force, respectively $(i=1,2,3,4$ refer to the left front wheel, right front wheel, left rear wheel, and right rear wheel); $C_{D}$ is the coefficient of air resistance; $\rho$ is the density of the air; a and $b$ are the distance between front and rear axial and vehicle centroid, respectively; $t_{f}$ and $t_{r}$ are tracks of front wheel and rear wheel, respectively; $\delta$ is the front wheel steering angle (assuming the steering angles of both front wheels are the same); Iz is the moment of inertia with respect to the roll axis; $A$ the longitudinal windward area of vehicle.

2.2. Wheel Model. This work mainly studied parameter estimation. Taking algorithm complexity, timelines, and model accuracy into consideration, Dugoff tire model was used. Longitudinal force and lateral force of tire are expressed with the following formula:

$$
\begin{aligned}
F_{x} & =\mu F_{z} C_{x} \frac{s}{1-s} f(L) \\
F_{y} & =\mu F_{z} C_{y} \frac{\tan \alpha}{1-s} f(L) \\
f(L) & = \begin{cases}(2-L) L, & L<1 \\
1, & L \geq 1\end{cases} \\
L & =\frac{(1-s)\left(1-\varepsilon v_{x} \sqrt{\left(C_{x} s\right)^{2}+\left(C_{y} \tan \alpha\right)^{2}}\right)}{2 \sqrt{\left(C_{x} s\right)^{2}+\left(C_{y} \tan \alpha\right)^{2}}}
\end{aligned}
$$

where $C_{x}$ is the longitudinal rigidity of tire; $C_{y}$ is the cornering stiffness of tire; $\alpha$ is the slip angle of tire; $s$ is the longitudinal slip (shift) rate of tire; when boundary value $L>1$, the tire is in the linear interval; when boundary value $L \leq 1$, the tire is not in the linear interval; $\varepsilon$ is the influential factor of speed which corrects the effect of sliding velocity on tire force; $\mu$ is the tire-road friction coefficient; $F_{z}$ is the vertical load of tire.

Here, $C_{x}$ and $C_{y}$ can be acquired by searching the table; $\alpha$ and $s$ can be expressed as functions of parameters $\delta, v_{x}$, $v_{y}, \omega_{i}, a_{x}$, and $a_{y}$ (see the specific equations in [25]). For the convenience of realizing estimation algorithm, Formula (5) can be simplified as the following normalized form:

$$
\begin{aligned}
& F_{x}=\mu F_{x}^{0}=\mu F_{z} C_{x} \frac{s}{1-s} f(L) \\
& F_{y}=\mu F_{y}^{0}=\mu F_{z} C_{y} \frac{\tan \alpha}{1-s} f(L)
\end{aligned}
$$

where $F_{x}{ }^{0}$ and $F_{y}{ }^{0}$ are normalized force of longitudinal and lateral tires, respectively, which are irrelevant with tire-road friction coefficient.

\section{Limited-Memory Adaptive Extended Kalman Filter}

3.1. Design of Estimator. For normal nonlinear system, the estimation model of status can be expressed with the following state-space equation:

$$
\begin{aligned}
& x(\mathrm{t})=f(x(t), u(t))+w(t) \\
& z(t)=h(x(t), u(t))+v(t)
\end{aligned}
$$

where the random variables $\boldsymbol{w}_{k} \in \mathrm{R}^{n}$ and $\boldsymbol{v}_{k} \in \mathrm{R}^{m}$ are process noise and measurement noise that are independent of each other with Gaussian distribution $\mathrm{p}(\mathrm{w}) \propto \mathrm{N}(0, \mathrm{Q})$ and $\mathrm{p}(\mathrm{v}) \propto$ $\mathrm{N}(0, \mathrm{R})$, with $\mathrm{Q} \in \mathrm{R}^{\mathrm{n} \times \mathrm{n}}$ and $\mathrm{R} \in \mathrm{R}^{\mathrm{m} \times \mathrm{m}}$ being the process and measurement covariance matrices, respectively.

This work mainly studied the real-time estimation of tireroad friction coefficient. With the friction coefficients of four wheels as the state variables of system, $\boldsymbol{x}=\left[\mu_{1}, \mu_{2}, \mu_{3}, \mu_{4}\right]^{T}$ was defined. With axial acceleration, lateral acceleration, and yaw rate of car measured by sensor (gyroscope) as observational variables of system, $z=\left[a_{x}, a_{y}, r\right]^{T}$ was defined. With front wheel steering angle and longitudinal and lateral normalized force of each wheel as input variables for control, $\boldsymbol{u}=(\delta$, $\left.F_{x i}{ }^{0}, F_{y i}{ }^{0}\right)^{T}$ was defined. By simultaneous Formulas (1)-(8), the following state formula and observation formula can be obtained: 


$$
\begin{aligned}
& x(t)=\phi x+w(t)=\left[\begin{array}{llll}
1 & 0 & 0 & 0 \\
0 & 1 & 0 & 0 \\
0 & 0 & 1 & 0 \\
0 & 0 & 0 & 1
\end{array}\right]\left[\begin{array}{l}
\mu_{1} \\
\mu_{2} \\
\mu_{3} \\
\mu_{4}
\end{array}\right]+w(t) \\
& z(t)=H x+\psi F_{w}+v(t)=\left[\begin{array}{llll}
\frac{F_{x 1}^{0} \cos \delta-F_{y 1}^{0} \sin \delta}{m} & \frac{F_{x 2}^{0} \cos \delta-F_{y 2}^{0} \sin \delta}{m} & \frac{F_{x 3}^{0}}{m} & \frac{F_{x 4}^{0}}{m} \\
\frac{F_{x 1}^{0} \sin \delta+F_{y 1}^{0} \cos \delta}{m} & \frac{F_{x 2}^{0} \sin \delta+F_{y 2}^{0} \cos \delta}{m} & \frac{F_{y 3}^{0}}{m} & \frac{F_{y 3}^{0}}{m} \\
H(3,1) & H(3,2) & H(3,3) & H(3,4)
\end{array}\right] \cdot\left[\begin{array}{l}
\mu_{1} \\
\mu_{2} \\
\mu_{3} \\
\mu_{4}
\end{array}\right]+\left[\begin{array}{l}
1 \\
0 \\
0
\end{array}\right] F_{w}+v(t)
\end{aligned}
$$

where

$$
\begin{aligned}
H(3,1)= & \frac{a\left(F_{x 1}^{0} \sin \delta+F_{y 1}^{0} \cos \delta\right)}{I_{z}} \\
& -\frac{t_{f} / 2\left(F_{x 1}^{0} \cos \delta-F_{y 1}^{0} \sin \delta\right)}{I_{z}} \\
H(3,2)= & \frac{a\left(F_{x 2}^{0} \sin \delta+F_{y 2}^{0} \cos \delta\right)}{I_{z}} \\
& +\frac{t_{f} / 2\left(F_{x 2}^{0} \cos \delta-F_{y 2}^{0} \sin \delta\right)}{I_{z}} \\
H(3,3)= & \frac{-\left(b F_{y 3}^{0}+t_{r} / 2 F_{x 3}^{0}\right)}{I_{z}} \\
H(3,4)= & \frac{-\left(b F_{y 4}^{0}-t_{r} / 2 F_{x 4}^{0}\right)}{I_{z}}
\end{aligned}
$$

where $\phi$ is state-transition matrix; $H$ is the linear Jacobian matrix; $\psi$ is the input matrix for control.

\subsection{EKF Algorithm of Limited Memory. As standard KF,} traditional EKF is also an infinitely increasing memory filter. When making the optimal estimation at the moment $k$, all the data before the moment $k$ should be used [26]. It means that, with the increase of $k$, the proportion of old data in filter will become larger, while that of new data is smaller. When there is model error and unknown time varying noise, the effect of new observation data is too small in correcting state estimation. Therefore, the effects on state estimation cannot be inhibited effectively, causing error accumulated to excessive large and even divergence [27]. For filter divergence caused by model error, the effect of new observation data should be enlarged to reduce the effect of old data on filter value.

In driving process, it is crucial to accurately estimate the current tire-road friction coefficient in real time for calculating tire force and developing the strategy for controlling vehicle stability. Particularly, old estimation may be not removed effectively in time, and the weight of new measurement may enlarge when estimating coefficient in sudden changes. At this time, the state estimation may have a large error. Such inherent error of model will certainly affect the estimation of tire-road friction coefficient in nonlinear condition. Therefore, the effects of current observation data should be further enlarged.

With limited-memory filter, only the first N-1 observation values before the current moment will be used to calculate the optimal estimation. In other words, the effects of old data before the N-1th observation value can be completely avoided. The timeliness of measurement data can be ensured by confirming the memory length $\mathrm{N}$ according to previous knowledge. By combining limited-memory filter with EFK algorithm, the accumulated error of old observation data can be eliminated to further deduce the extended Kalman filter formula with limited memory:

(1) Further prediction of system state:

$$
{\widehat{X_{N}(k, k-1)}}=\phi_{(k, k-1)}{\widehat{X_{N}(k-1, k-1)}}_{\psi}+\psi U_{(k)}
$$

(2) Further prediction of error variance matrix:

$$
P_{N(k, k-1)}=\phi_{(k, k-1)} P_{N(k-1, k-1)} \phi_{(k, k-1)}^{T}+Q_{k-1}
$$

(3) Gain matrix:

$$
\begin{aligned}
& K_{(k)}=P_{N(k, k)} H_{(k)}^{T} R_{k}^{-1}, \\
& \overline{K_{(k)}}=K_{(k-N)}=P_{N(k, k)} \phi_{(k-N, k)}^{T} H_{(k-N)}^{T} R_{k-N}^{-1}
\end{aligned}
$$

(4) Composition error at moment $k-N$ :

$$
\begin{aligned}
& R_{k-N}^{\tau-1} \\
& \quad=R_{k-N}^{-1} \\
& \quad+H_{(k-N)}\left\{\sum_{l=k-N+1}^{k}\left(\phi_{l-1} Q_{l-1} \phi_{l-1}^{T}\right)\right\} H^{T}{ }_{(k-N)}
\end{aligned}
$$

(5) Residual sequence:

$$
\begin{aligned}
\widetilde{Z_{(k)}} & =Z_{(k)}-H_{(k)} \phi_{(k, k-1)} \widehat{X_{N}(k, k-1)}, \\
\widetilde{Z_{(k-N)}} & =Z_{(k-N)}-H_{(k-N)} \phi_{(k-N, k)} \phi_{(k, k-1)} \widehat{X_{N}(k, k-1)}
\end{aligned}
$$


(6) Optimal estimation of system:

$$
\widehat{X}_{N(k, k)}=\widehat{X}_{N(k, k-1)}+K_{(k)} \widetilde{Z_{(k)}}-K_{(k-N)} \widetilde{Z_{(k-N)}}
$$

(7) Error variance matrix of system:

$$
\begin{aligned}
& P_{N(k, k)}=\left[P_{N}^{-1}{ }_{(k, k-1)}+H_{(k)}^{T} R_{k}^{-1} H_{(k)}\right. \\
& \left.-\phi_{(k-N, k)}^{T} H_{(k-N)}^{T} R_{k-N}^{\tau-1} H_{(k-N)} \phi_{(k-N, k)}\right]^{-1}
\end{aligned}
$$

where $Q$ and $R$ are system noise and observation noise, respectively; $N$ is the length of limited memory.

When $k \leq N$, the observation time $k$ is less than memory length $N$, and only the extended Kalman formula can be used without limited-memory filter. The calculation starts from the initial values $\widehat{X}(0,0)=E\{X(0)\}$ and $P(0,0)=\operatorname{var}\{X(0)\}$ to values $\widehat{X}(N, N)$ and $P(N, N)$. However, starting from $k$ $=N+1$, the limited-memory filter $\widehat{X_{(N)}}(k, k)$ will be affected by $\widehat{X}(0,0)$ and $P(0,0)$ when taking $\overline{X_{(N)}}(N, N)=\widehat{X}(N, N)$ and $P_{(N)}(N, N)=P(N, N)$ as initial values for limitedmemory filtering. This does not satisfy limited-memory filter. Therefore, effects of initial values $\widehat{X}(0,0)$ and $P(0,0)$ on $\widehat{X_{(N)}}(k, k)(k>N)$ should be avoided. When setting $k \leq N$, let $H_{(k-N)}=0$. At this time, $\widehat{X_{(N)}}(k, k)$ is the filter of EKF. When $k>N$, let $P_{(0,0)}^{-1}=0, \widehat{X}(0,0)=0$.

3.3. Fuzzy Kalman Filter Algorithm. In actual vehicle systems, process noise and measurement noise are constantly changing. The process noise is affected by factors such as the operating conditions of the vehicle itself and environmental conditions. The measurement noise is affected by the accuracy of the sensor and the environmental conditions of the measurement. Vehicles are highly nonlinear systems, and the actual operating conditions of the vehicle are complicated, which makes it difficult to predict and accurately measure vehicle noise. Since the vehicles studied mainly operate in conventional urban working conditions, process noise can be determined by the statistical characteristics of noise obtained through experiments. In this case, we assume that process noise can be considered to be a constant value. On the other hand, considering the accuracy of the acceleration sensor owned by the actual vehicle, it is considered that the measurement noise has a greater impact on the vehicle system we are currently testing. Therefore, it is necessary to focus on eliminating the adverse effect of measurement noise.

In actual driving, the statistical characteristics of observation noise are changing. Covariance matrix of measured noise $R_{k}$ is dynamically adjusted by fuzzy interference system to realize the self-adaptive regulation of filter [28]. The work defines the observation noise variance $R_{k}$ at moment $k$, making it satisfy $\widehat{R_{\mathrm{k}}}=\widehat{R_{\mathrm{k}-1}}+\Delta R$.

The specific steps of self-adaptive adjustment of fuzzy Kalman are as follows.

(1) Calculate the theoretical value of residual covariance in extended Kalman filter algorithm:

$$
\begin{aligned}
K_{(k)} & =P_{N(k, k)} H_{(k)}^{T} R_{k}^{-1} \\
P_{(k)} & =\left[I_{(\mathrm{n})}-K_{(\mathrm{k})} H_{(k)}\right] P_{(k-1)}
\end{aligned}
$$

That is,

$$
P_{(k)}=\left[I_{(\mathrm{n})}-P_{N(k, k)} H_{(k)}^{T} R_{k}^{-1} H_{(k)}\right] P_{(k-1)}
$$

(2) Appropriately calculate the actual residual covariance. Residual error is $r_{k}=z_{k}-H_{k}^{T} x_{k / k-1}$ and the mean value is $\bar{r}=(1 / n) \sum_{j=k-n+1}^{k} r_{j}$, so the actual residual variance is $C_{k}=$ $(1 / n) \sum_{j=k-n+1}^{k} r_{j} r_{j}^{T}$.

(3) Use fuzzy interference engine for self-adaptive adjustment of $r_{k}$. Define the matching rate e $(\mathrm{k})$ between variants $P_{z}$ and $C_{k}$ as $e(\mathrm{k})=P_{z}-C_{k}$.

In fuzzy control machine, the difference $\mathrm{e}(\mathrm{k})$ between actual variance and theoretical variance is taken as input and the regulating variable of measurement $\Delta R$ as output. The adjustment amount of $\Delta R$ is obtained by repeating a large number of experiments. Figure 2 shows the subordinating degree functions of input and output.

The fuzzy subsets of input and output are defined as follows [29]:

(1) If $e(\mathrm{k}) \approx 0$ (which means the actual variance is close to theoretical variance), $r_{k}$ keeps constant; that is, if $(\mathrm{k}) \in \mathrm{ZE}$, then $\Delta R \in M$.

(2) If $e(\mathrm{k})<0$ (which means the actual variance is larger than theoretical variance), reduce $r_{k}$; that is, if $e(\mathrm{k}) \in N$, then $\Delta R \in D$.

(3) If $e(\mathrm{k})>0$ (which means the actual variance is less than theoretical variance), increase $r_{k}$; that is, if $e(\mathrm{k}) \in P$, then $\Delta R \in I$.

The defuzzification method used in this method is the centroid method.

\section{Conclusions}

All experimental maneuvers are driven with a type of allwheel drive vehicle. Using an inertial measurement unit, precise measurements of yaw-rate, sideslip angle, longitudinal and lateral accelerations, and velocity of the c.o.g. are taken. The measurements of the wheel's rotational velocity are obtained reading out the CAN bus. To test the validity of designed estimation algorithm, Matlab/Simulink and Carsim software were used for joint simulation experience.

The system status variable was initially set as $\boldsymbol{x}=$ $\left[\begin{array}{llll}0.001 & 0.001 & 0.001 & 0.001\end{array}\right]^{T}$ in the beginning of simulation, while the covariance matrices are initialized as

$$
\begin{aligned}
& \mathrm{Q}_{0}=\operatorname{diag}\left[10^{-9} ; 10^{-9} ; 10^{-9} ; 10^{-9}\right], \\
& \mathrm{P}_{0}=\operatorname{diag}\left[10^{-6} ; 10^{-6} ; 10^{-6} ; 10^{-6}\right], \\
& \mathrm{R}_{0}=\operatorname{diag}\left[10^{-4} ; 10^{-4} ; 10^{-4}\right] .
\end{aligned}
$$

The longitudinal and lateral accelerated speed and yaw rate output by Carsim were taken as measurement values of system with Gaussian white noise; corner of steering wheel and normalized force of each wheel were taken as the control input to system. Then, the current tire-road friction coefficient was estimated in real time; LM-AEKF estimation and traditional EKF estimation were compared with the output of Carsim. 


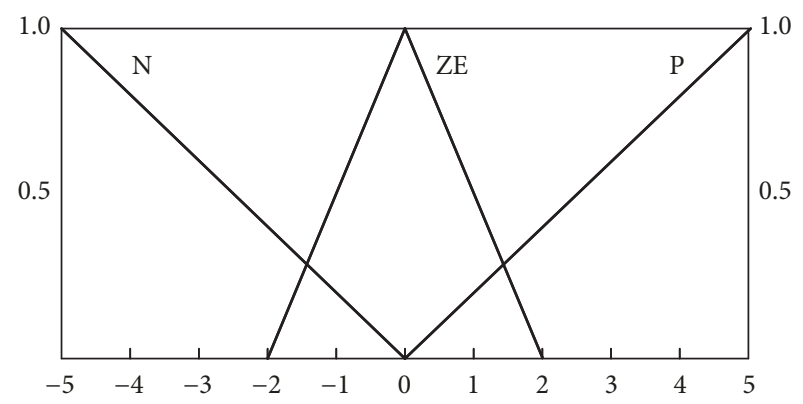

(a) Subordinating degree functions of input

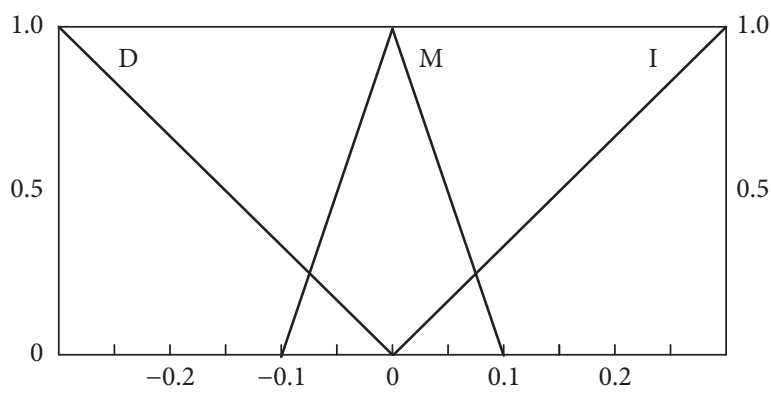

(b) Subordinating degree functions of output

FIGURE 2: The subordinating degree functions of input and output.

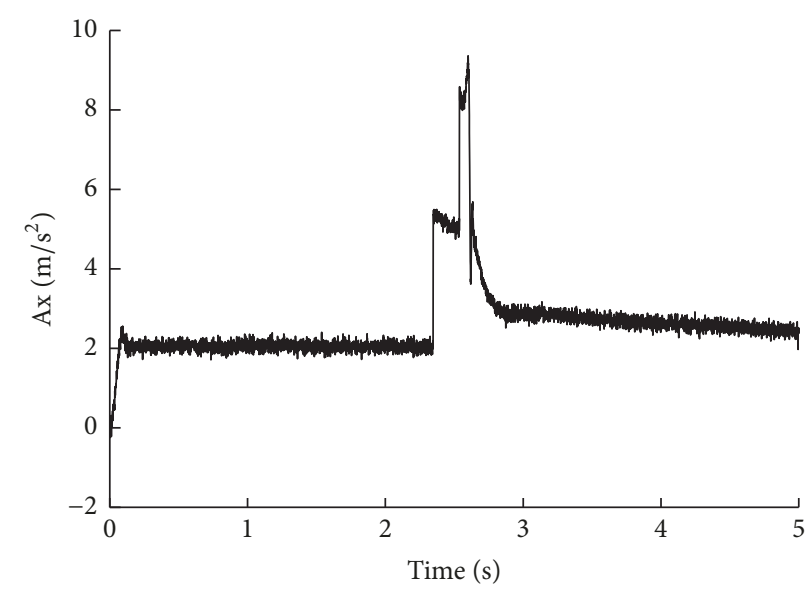

FIgURE 3: The longitudinal accelerated measurement with Gaussian white noise.

4.1. Docking Road Test. The acceleration test with starting speed of $30 \mathrm{~km} / \mathrm{h}$ was set for a vehicle on the road of sudden change with tire-road coefficient between 0.2 and 0.8 in Carsim. The road length with tire-road friction coefficient of 0.2 is 25 meters; the openness of accelerator pedal is $50 \%$; the steering wheel angle in test keeps 0 .

Figure 3 shows the longitudinal accelerated measurement with Gaussian white noise. Figure 4 shows the estimation of tire-road friction coefficient by traditional EKF algorithm, UKF algorithm, and LM-AEKF algorithm.

According to simulation results, both algorithms have good estimation effects before the sudden change of tireroad friction coefficient. However, when the car enters new road, severe buffeting occurs to estimation by traditional EKF algorithm. Moreover, the estimation of tire-road friction coefficient tends to have larger error and even be convergent with time. Compared with EKF algorithm, UKF algorithm estimation results have less fluctuation, and the results tend to converge, which can reach the steady-state value more quickly. On the contrary, LM-AEKF algorithm not only eliminates the buffeting in estimation, but also rapidly converges the real value. Therefore, LM-AEKF has better accuracy and stability.

4.2. Double Lane Change Test. The double lane change test with constant speed of $85 \mathrm{~km} / \mathrm{h}$ was set for a vehicle on the road with tire-road coefficient of 0.8 in Carsim. Figures 5 and 6 show the measurement of lateral accelerated speed and yaw rate with Gaussian white noise, respectively. Figure 7 shows the estimation of tire-road friction coefficient by traditional EKF algorithm, UKF algorithm, and LM-AEKF algorithm. According to simulation results, the vehicle is in the nonlinear area at about $4 \mathrm{~s}$. By traditional EKF algorithm, the estimation greatly changes with the most severe buffeting near nonlinear area. Converging slowly, there is a large difference between stable value and real value. UKF estimation results also show obvious fluctuations. However, compared with EKF algorithm, UKF estimation results can rapidly converge and reach steady-state values more quickly. On the contrary, by LM-AEKF algorithm, the estimation converges rapidly, greatly improving the overall accuracy and stability.

4.3. Bisectional Road Test. In Carsim, the vehicle was driven straightly on a flat bisectional road at a uniform speed of 40 $\mathrm{km} / \mathrm{h}$, wherein the roadside adhesion coefficients of the left and right sides were 0.2 and 0.8 , respectively.

Figure 8 shows the measurement of longitudinal accelerated speed with Gaussian white noise. Figures 9 and 10 respectively show the estimation of tire-road friction coefficient by traditional EKF algorithm, UKF algorithm and LMAEKF algorithm. Among them, the left wheels are driven on a road surface with a tire-road friction coefficient of 0.2 , and 


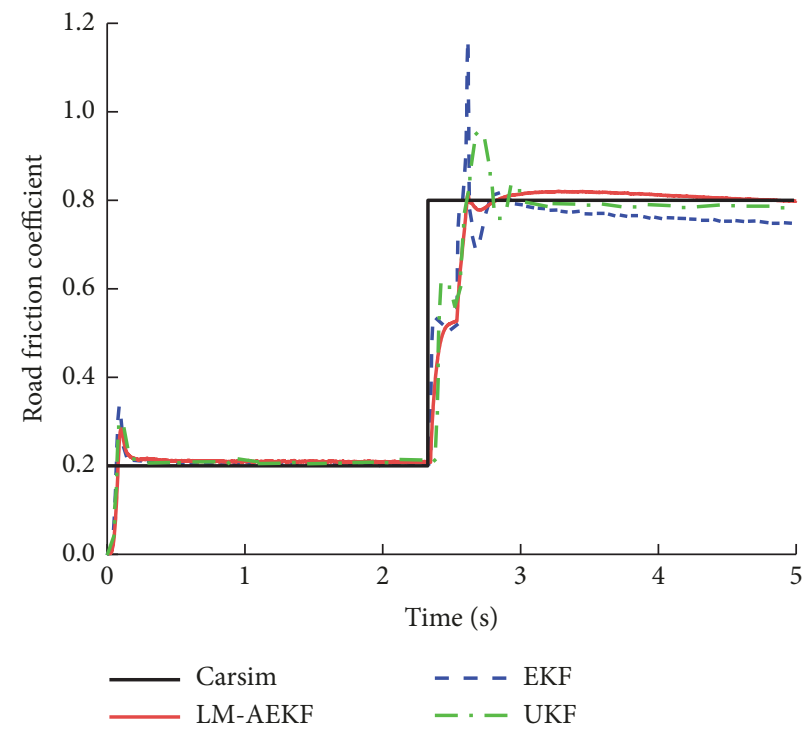

FIGURE 4: The estimation of tire-road friction coefficient.

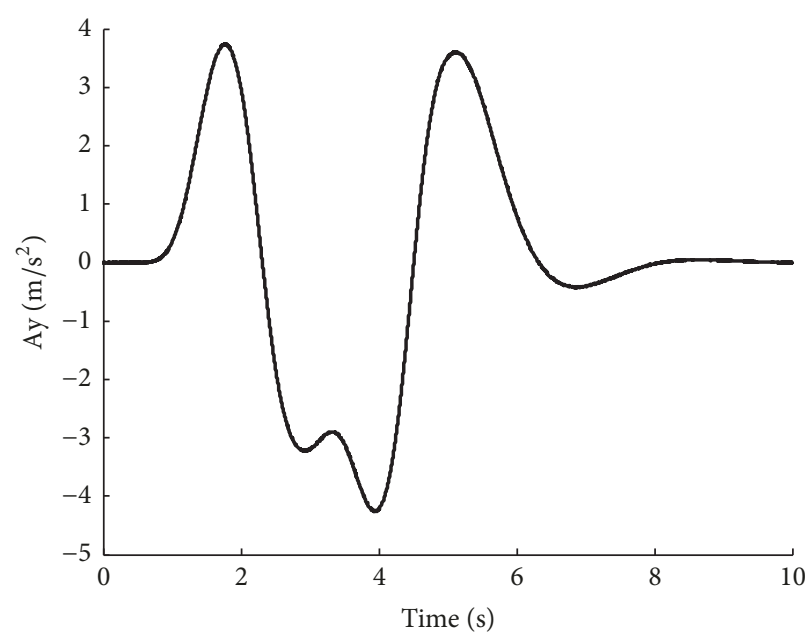

FIGURE 5: Lateral accelerated speed with Gaussian white noise.

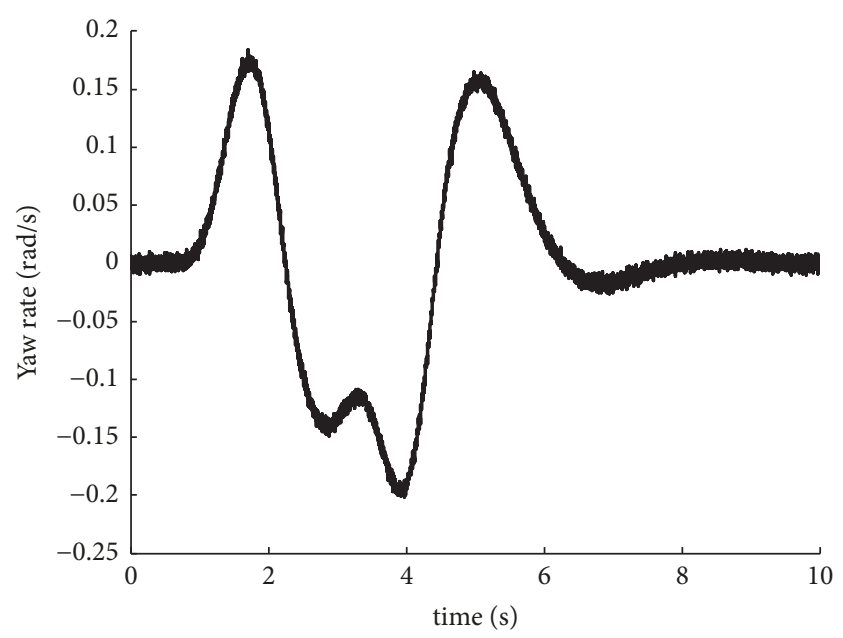

FIgURE 6: Yaw rate with Gaussian white noise. 


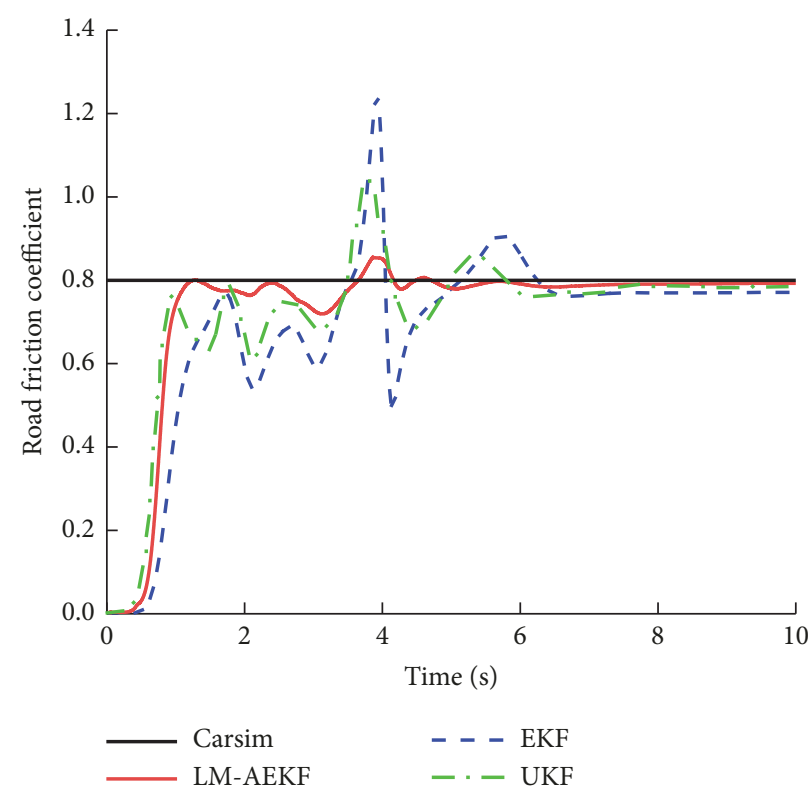

FIGURE 7: The estimation of tire-road friction coefficient.

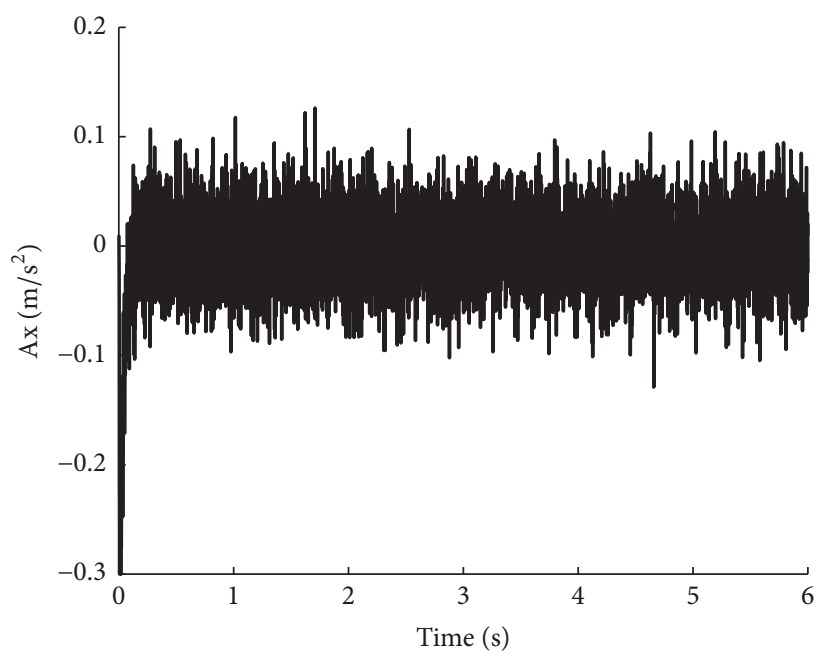

FIGURE 8: The longitudinal accelerated measurement with Gaussian white noise.

the right wheels are driven on a road surface with a tire-road friction coefficient of 0.8 According to simulation results, by traditional EKF algorithm, the estimation results greatly change with the most severe buffeting and converge slowly. Obviously, there is a large difference between stable value and real value. By UKF algorithm, the estimation value can approach the steady-state value more quickly, the vibration amplitude is relatively gentle, and the effect is improved. On the contrary, by LM-AEKF algorithm, the estimation converges rapidly, greatly improving the overall accuracy and stability.

\section{Vehicle Test}

To further verify the effectiveness of LM-AEKF algorithm to tire-road friction coefficient estimation, this work conducted vehicle test based on a type of four-wheel independent drive light-duty off-road vehicle. Steering wheel angle, rotational speed of each tire, and longitudinal velocity required in this test were collected from the sensor of steering wheel angle, sensor of wheel speed, and GPS. In addition, the longitudinal and lateral accelerated speeds and yaw rate were collected by gyroscope in sampling time of $10 \mathrm{~ms}$. Lateral speed cannot be directly measured, while error accumulation may be caused in direct measurement of lateral accelerated speed. Therefore, standard Kalman filter processing was used for lateral accelerated speed in this work. Above values measured with instrument can be loaded to CAN bus and transferred to upper computer software, controldesk, through D/A model of $\mathrm{dSPACE}$ by I/O interface. Therefore, the data monitored in real time can be collected and saved. Figures 11 and 12 show the testing vehicle, equipment, and data processing flow. 


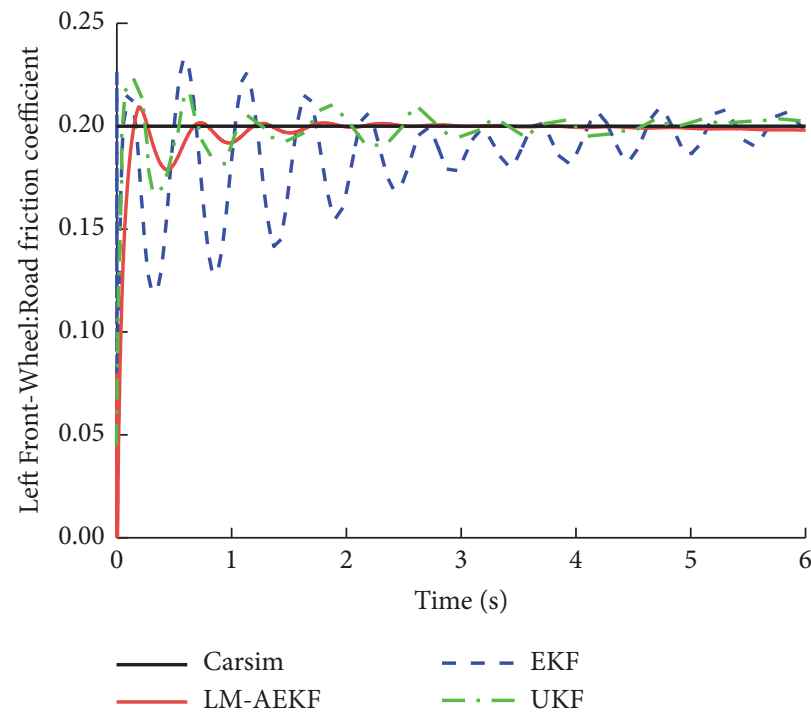

FIGURE 9: The estimation of tire-road friction coefficient of left front-wheel.

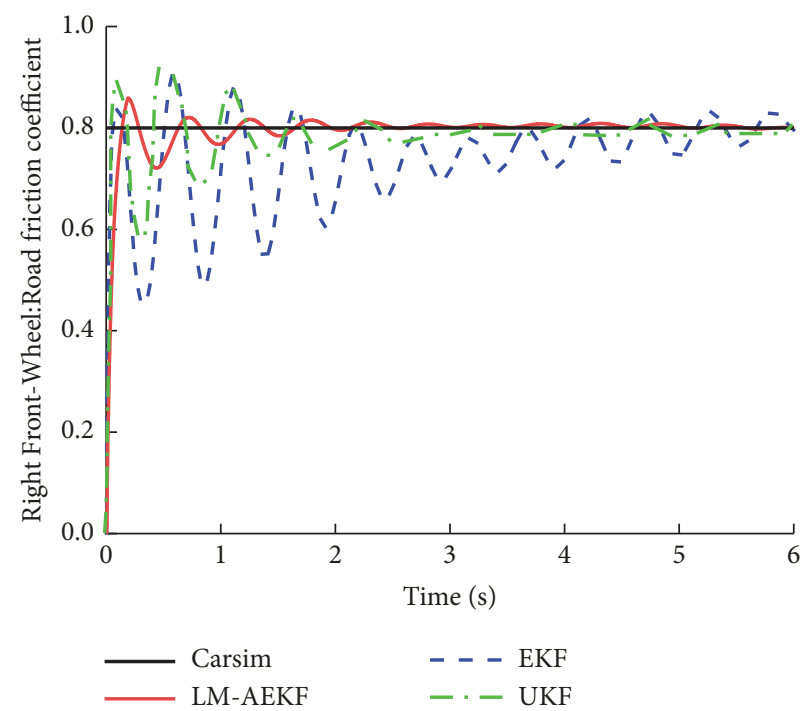

Figure 10: The estimation of tire-road friction coefficient of right front-wheel.

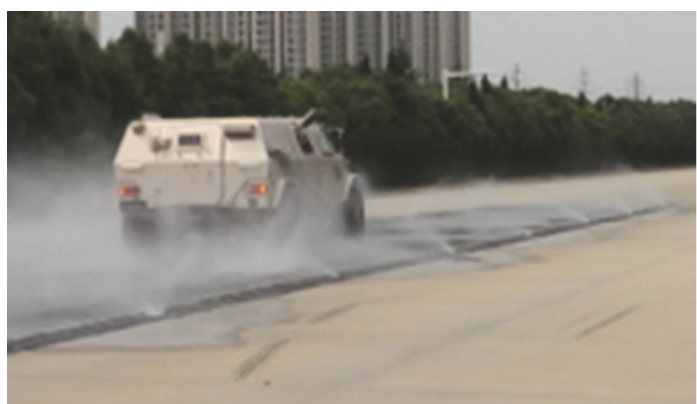

Figure 11: The testing vehicle. 


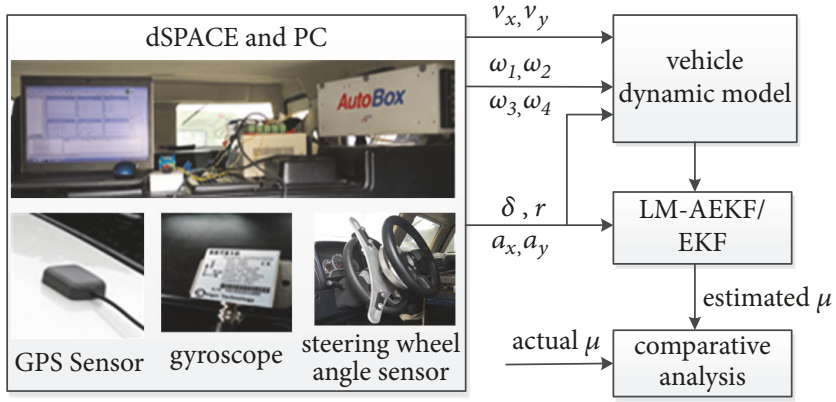

FIGURE 12: The testing equipment and data processing flow.

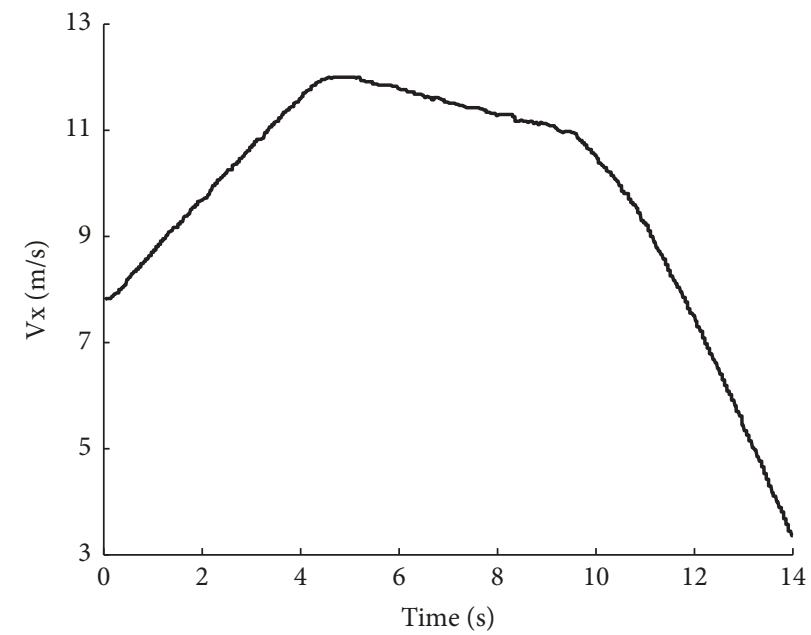

FIGURE 13: Longitudinal velocity.

With information collected in the test, the designed LMAEKF algorithm can be realized in Matlab/Simulink. Then, the estimation by LM-AEKF algorithm can be compared with that by UKF algorithm and EKF algorithm.

5.1. Docking Road Test. For vehicle test, watering ABS road and dry, flat, and clean road of cement concrete were selected as low-friction road (the friction coefficient is between 0.22 and 0.25 ) and high-friction road (the friction coefficient is between 0.78 and 0.8 ), respectively, in standard test site (see Figure 11 for road test). To take advantage of road conditions and highlight the conditional adaptation of algorithms, low-friction acceleration test and low-friction and high-friction sliding test were continuously conducted for testing vehicle. Figures 13-15 show the longitudinal velocity, longitudinal acceleration, wheel speed, and other information.

Figure 16 shows the estimation of algorithms. It can be seen that the estimation results of UKF algorithm and LMAEKF algorithm are generally stable and accurate. After the vehicle entered high-friction road at about $10 \mathrm{~s}$, UKF algorithm and LM-AEKF algorithm responded rapidly, showing that both two algorithms have good adaptation to sudden changes. On the contrary, tire-road friction coefficient estimated by traditional EKF algorithm is less stable for the slower response to sudden changes. After meeting new

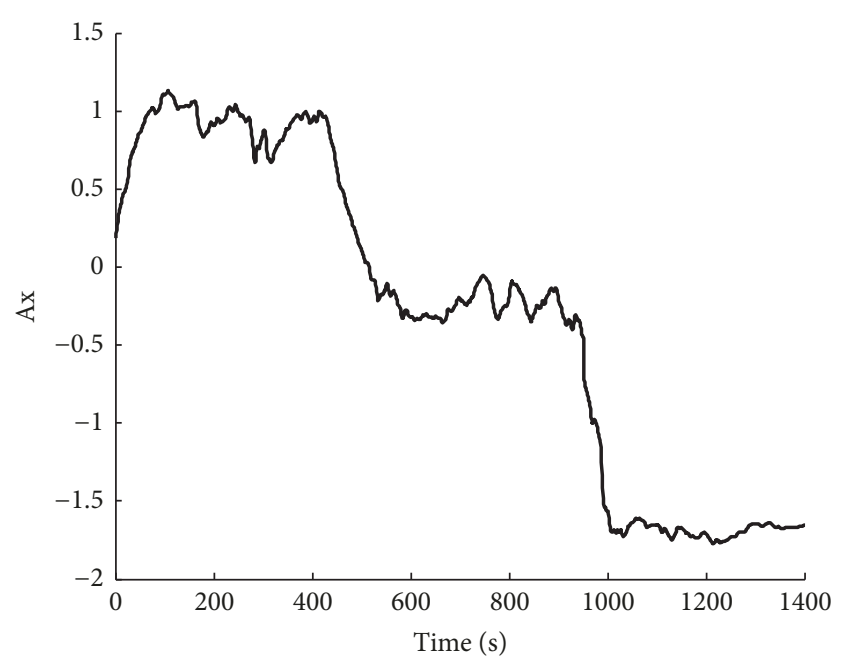

FIGURE 14: Longitudinal acceleration.

conditions, the difference between convergence and actual value tends to increase because of the accumulated error.

5.2. Double Lane Change Test. Double lane change test was conducted for vehicle at constant speed according to ISO 3888-1-1999 on the horizontal dry asphalt road in test 


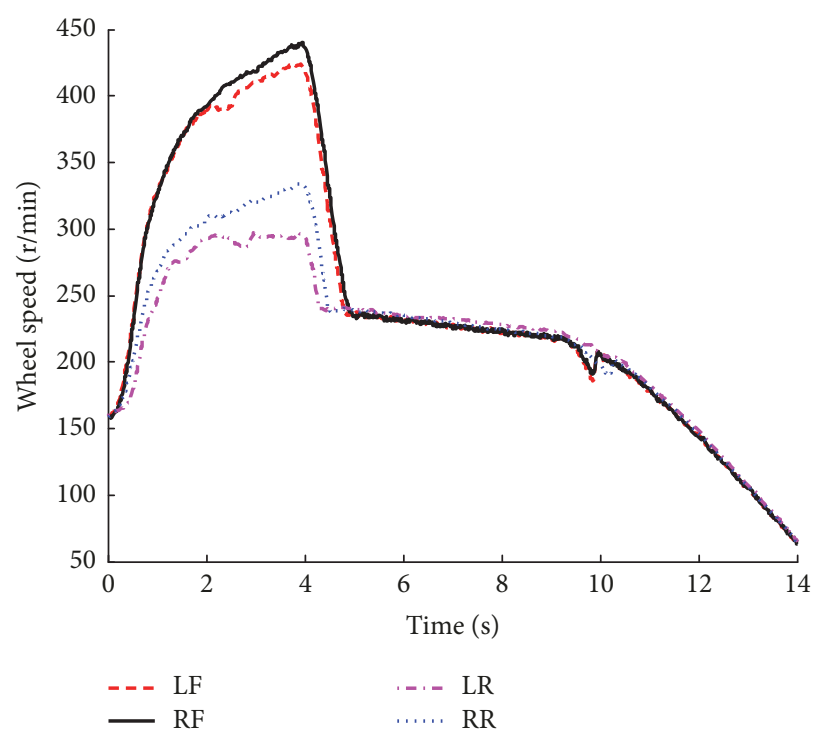

FIGURE 15: Wheel speed.

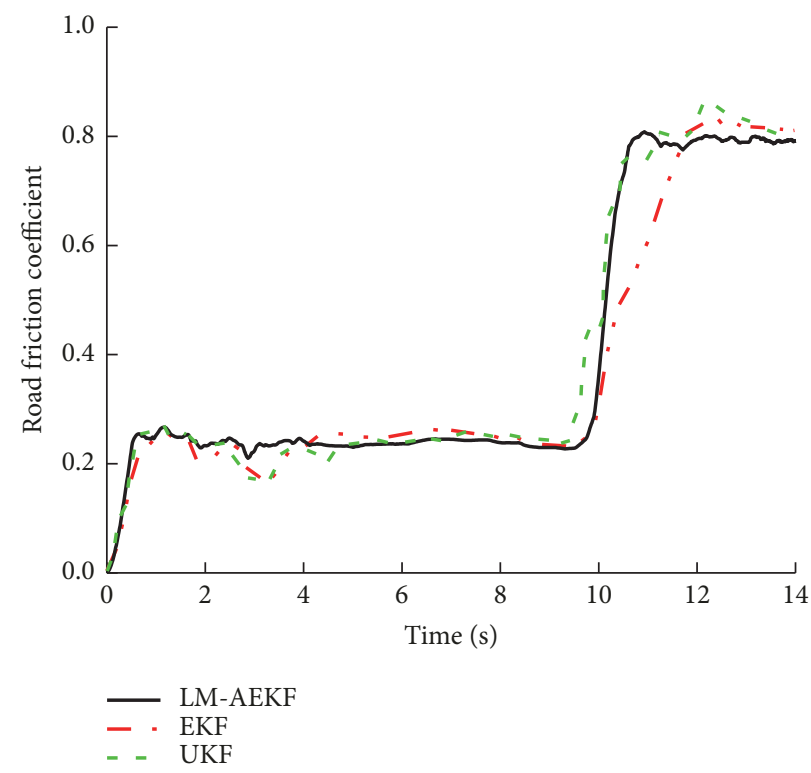

FIGURE 16: The estimation of tire-road friction coefficient.

field (with friction coefficient of about 0.8) [30]. Considering the security, the testing speed was $45 \mathrm{~km} / \mathrm{h}( \pm 1 \mathrm{~km} / \mathrm{h})$. Figures 17-19 show some measurements recorded by GPS and gyroscope. Figure 20 shows the estimation of tire-road friction coefficient. According to the test results, tire-road friction coefficients estimated by UKF algorithm and LMAEKF algorithm are stable with fast convergence and high accuracy. However, in the initial stage of estimation, the fluctuation of the UKF algorithm is more obvious than the LM-AEKF algorithm, and the convergence speed of the UKF algorithm is also slightly slower, but the steady-state value of the UKF estimation result approaches the actual value. On the contrary, the coefficient estimated by traditional EKF algorithm changes greatly with slow convergence and some difference from actual value.

\section{Conclusions}

Vehicle model has errors and cannot obtain statistical characteristics of time-varying noise. Therefore, this work proposed EKF algorithm that can fade memory by exponential weighting based on traditional EKF algorithm. Then, tireroad friction coefficient was estimated in real time with this algorithm. By comparing the results of simulation tests by two algorithms in different conditions, conclusions are drawn as follows: 


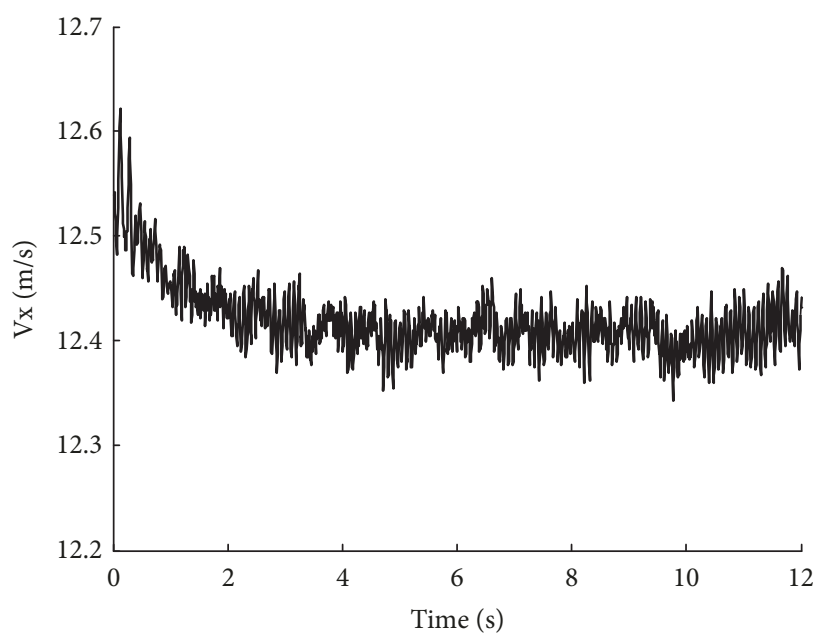

FIGURE 17: Longitudinal velocity.

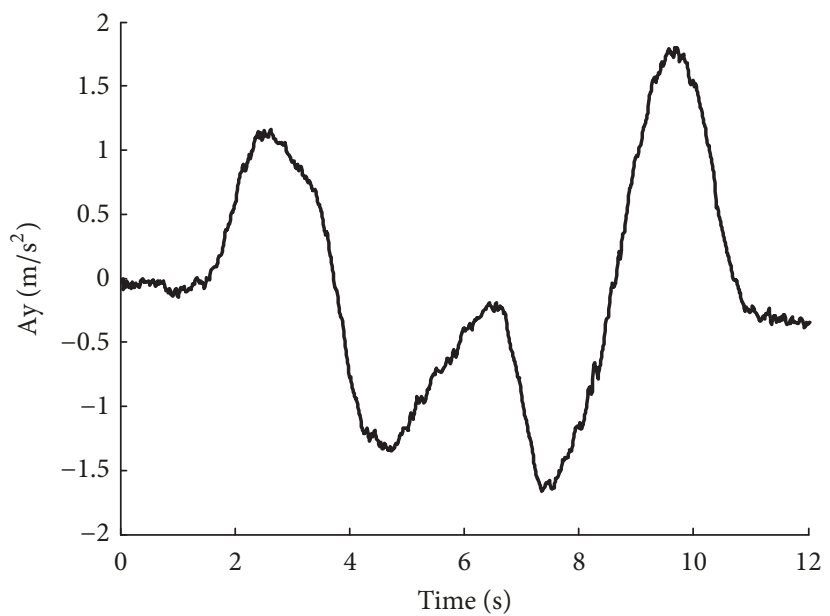

FIGURE 18: Lateral acceleration.

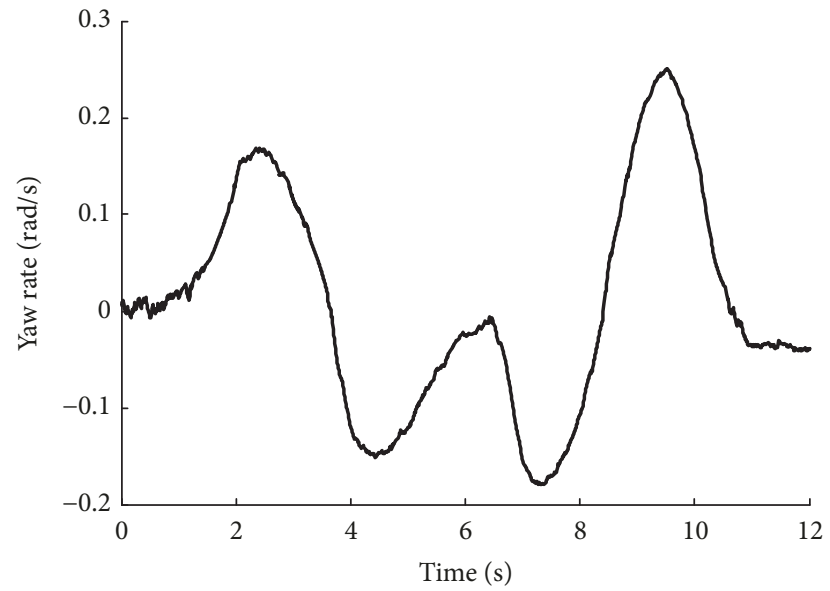

Figure 19: Yaw rate. 


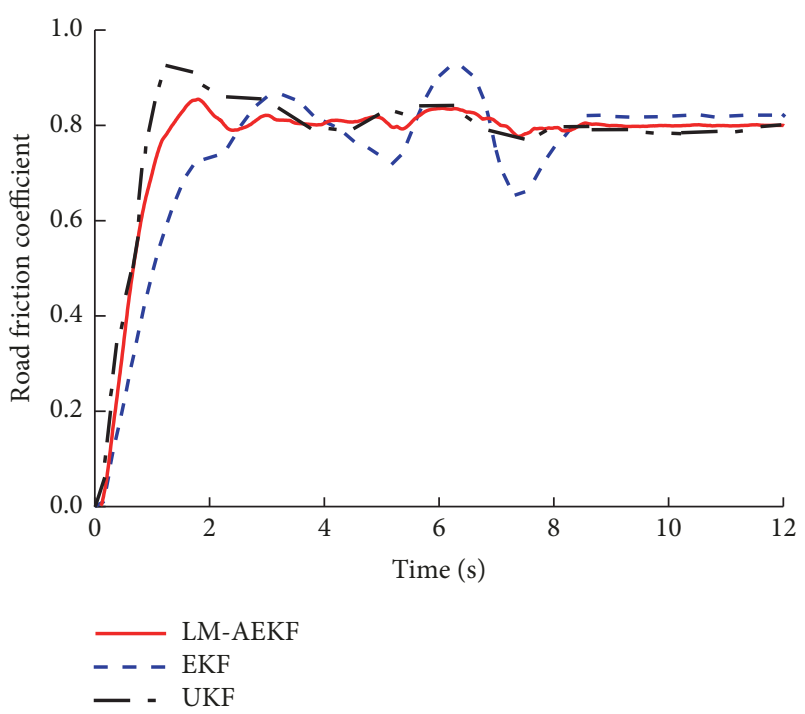

FIGURE 20: The estimation of tire-road friction coefficient.

(a) The algorithm proposed in this work can better estimate current tire-road friction coefficient in different conditions. In the same condition, the proposed algorithm has better estimation accuracy and stability with faster response speed than traditional EKF algorithm.

(b) LM-AEKF algorithm is easy to be operated without affecting timeliness. This algorithm has high estimation accuracy and filter stability even if the model has errors and noise statistics is unknown. It means that this algorithm is self-adaptive.

\section{Data Availability}

The data that support the findings of this study are available on request from the corresponding author. The data are not publicly available due to their containing information that could compromise the privacy of research participants.

\section{Conflicts of Interest}

The authors declare that they have no conflicts of interest.

\section{References}

[1] L. Li, H. Li, J. Song, C. Yang, and H. Wu, "Road friction estimation under complicated maneuver conditions for active yaw control," Chinese Journal of Mechanical Engineering, vol. 22, no. 4, pp. 514-520, 2009.

[2] L. Li, K. Yang, G. Jia, X. Ran, J. Song, and Z.-Q. Han, "Comprehensive tire-road friction coefficient estimation based on signal fusion method under complex maneuvering operations," Mechanical Systems and Signal Processing, vol. 56, pp. 259-276, 2015.

[3] K. Han, Y. Hwang, E. Lee, and S. Choi, "Robust estimation of maximum tire-road friction coefficient considering road surface irregularity," International Journal of Automotive Technology, vol. 17, no. 3, pp. 415-425, 2016.

[4] S. Khaleghian, A. Emami, and S. Taheri, "A technical survey on tire-road friction estimation," Friction, vol. 5, no. 2, pp. 123-146, 2017.
[5] B. S. Kanwar and T. Saied, "Estimation of tire-road friction coefficient and its application in chassis control systems," Systems Science \& Control Engineering, vol. 3, no. 1, pp. 39-61, 2015.

[6] Y. Q. Zhao, H. Q. Li, F. Lin, J. Wang, and X. W. Ji, "Estimation of road friction coefficient in different road conditions based on vehicle braking dynamics," Chinese Journal of Mechanical Engineering, vol. 6, no. 1, pp. 104-108, 2014.

[7] M. Wielitzka, M. Dagen, and T. Ortmaier, "State estimation of vehicle's lateral dynamics using unscented Kalman filter," in Proceedings of the 2014 53rd IEEE Annual Conference on Decision and Control, CDC 2014, pp. 5015-5020, December 2014.

[8] K. Li, X. Chen, and G. J. Zhou, "Complex motion modeling and state estimation in road coordinates," Journal of Harbin Institute of Technology, vol. 24, no. 1, pp. 19-25, 2017.

[9] L. Xu, X. R. Li, Z. Duan, and J. Lan, "Modeling and state estimation for dynamic systems with linear equality constraints," IEEE Transactions on Signal Processing, vol. 61, no. 11, pp. 2927-2939, 2013.

[10] M. Choi and S. Choi, "Model predictive control for vehicle yaw stability with practical concerns," IEEE Transactions on Vehicular Technology, vol. 63, no. 8, pp. 3539-3548, 2014.

[11] H. Kim, S. Lee, and J. K. Hedrick, "Active yaw control for handling performance improvement by using traction force," International Journal of Automotive Technology, vol. 16, no. 3, pp. 457-464, 2015.

[12] K. Enisz, D. Fodor, I. Szalay, and G. Kohlrusz, "Improvement of active safety systems by the extended Kalman filter based estimation of tire-road friction coefficient," in Proceedings of the 2014 IEEE International Electric Vehicle Conference, IEVC 2014, pp. 917-925, Florence, Italy, December 2014.

[13] Z. J. Zhou, C. H. Hu, B. C. Zhang, and L. Chen, "An improved fuzzy kalman filter for state estimation of nonlinear systems," International Journal of Systems Science, vol. 41, no. 5, pp. 537546, 2010.

[14] S. Konatowski, P. Kaniewski, and J. Matuszewski, "Comparison of estimation accuracy of EKF, UKF and PF filters," Annual of Navigation, vol. 23, no. 1, pp. 69-87, 2016.

[15] Y. F. Lian, Y. Zhao, L. L. Hu, and Y. T. Tian, "Cornering stiffness and sideslip angle estimation based on simplified lateral dynamic models for four-in-wheel-motor-driven electric vehicles with lateral tire force information," International Journal of Automotive Technology, vol. 16, no. 4, pp. 669-683, 2015.

[16] W. Mark, D. Matthias, and O. Tobias, "Joint unscented Kalman filter for state and parameter estimation in vehicle dynamics," in Proceedings of the IEEE Conference on Control Applications (CCA, DOI: 0.1109/CCA.2015.7320894, 2015.

[17] R. Rajamani, G. Phanomchoeng, D. Piyabongkarn, and J. Y. Lew, "Algorithms for real-time estimation of individual wheel tire-road friction coefficients," IEEE/ASME Transactions on Mechatronics, vol. 17, no. 6, pp. 1183-1195, 2012.

[18] M. Wielitzka, M. Dagen, and T. Ortmaier, "Sensitivitybased road friction estimation in vehicle dynamics using the unscented kalman filter," in Proceedings of the 2018 Annual American Control Conference, ACC 2018, pp. 2593-2598, June 2018.

[19] M. Wielitzka, M. Dagen, and T. Ortmaier, "Sensitivitybased road friction estimation in vehicle dynamics using the unscented kalman filter," in Proceedings of the 2018 Annual American Control Conference (ACC), pp. 2593-2598, Milwaukee, Wis, USA, June 2018. 
[20] X. J. Jin and G. Yin, "Estimation of lateral tire-road forces and sideslip angle for electric vehicles using interacting multiple model filter approach," Journal of The Franklin Institute, vol. 352, no. 2, pp. 686-707, 2015.

[21] X. Jin and G. Yin, "Estimation of lateral tire-road forces and sideslip angle for electric vehicles using interacting multiple model filter approach," Journal of The Franklin Institute, vol. 352, no. 2, pp. 686-707, 2015.

[22] S. Solmaz and S. Ç. Başlamişli, "Simultaneous estimation of road friction and sideslip angle based on switched multiple nonlinear observers," IET Control Theory \& Applications, vol. 6, no. 1, pp. 2235-2247, 2012.

[23] D. Iraj, R. Asghar, M. Mahdi, and A. Pouyan, "Identification of tire forces using dual unscented kalman filter algorithm," Nonlinear Dynamics, vol. 78, no. 3, pp. 1907-1919, 2014.

[24] I. Davoodabadi, A. Ramezani, M. Mahmoodi-k, and P. Ahmadizadeh, "Erratum to: identification of tire forces using dual unscented kalman filter algorithm," Nonlinear Dynamics, vol. 78, no. 4, p. 2985, 2014.

[25] J. Na, Y. Huang, X. Wu et al., "Active adaptive estimation and control for vehicle suspensions with prescribed performance," IEEE Transactions on Control Systems Technology, vol. 26, no. 6, pp. 1-15, 2017.

[26] A. H. Ahangarnejad and S. C. Başlamişli, "Adap-tyre: DEKF filtering for vehicle state estimation based on tyre parameter adaptation," International Journal of Vehicle Design, vol. 71, no. 1, pp. 52-74, 2016.

[27] J. Yang, J. Na, Y. Guo, and X. Wu, "Adaptive estimation of road gradient and vehicle parameters for vehicular systems," IET Control Theory \& Applications, vol. 9, no. 6, pp. 935-943, 2015.

[28] S. Najafi, G. W. Flintsch, and S. Khaleghian, "Fuzzy logic inference-based Pavement Friction Management and real-time slippery warning systems: A proof of concept study," Accident Analysis \& Prevention, vol. 90, no. 2, pp. 41-49, 2016.

[29] R. Jayachandran, S. D. Ashok, and S. Narayanan, "Fuzzy logic based modelling and simulation approach for the estimation of tire forces," Procedia Engineering, vol. 64, pp. 1109-1118, 2013.

[30] T. Goggia, A. Sorniotti, L. De Novellis et al., "Integral sliding mode for the torque-vectoring control of fully electric vehicles: Theoretical design and experimental assessment," IEEE Transactions on Vehicular Technology, vol. 64, no. 5, pp. 1701-1715, 2015. 


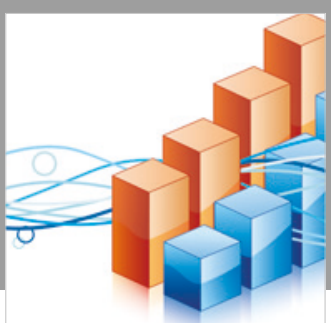

Advances in

Operations Research

\section{-n-m}
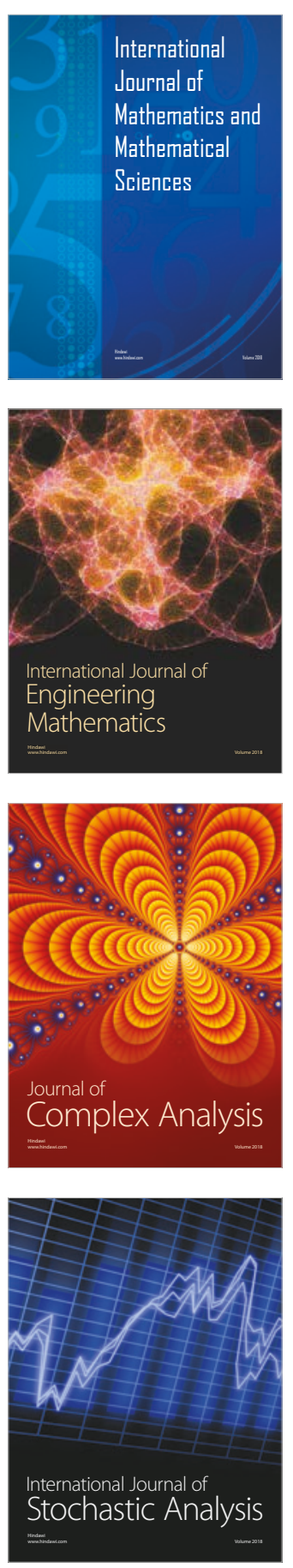
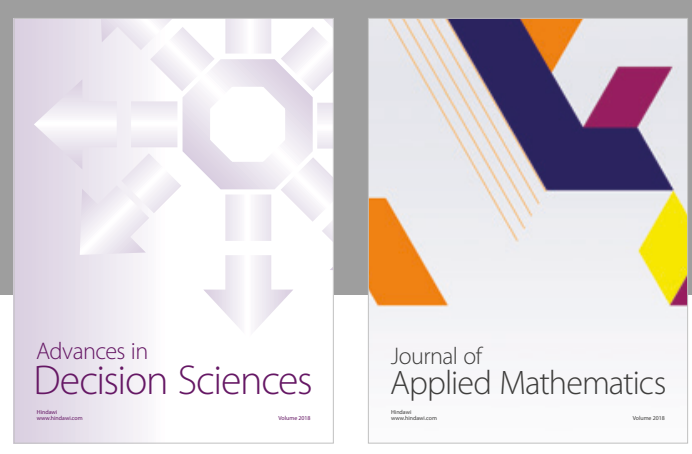

Journal of

Applied Mathematics
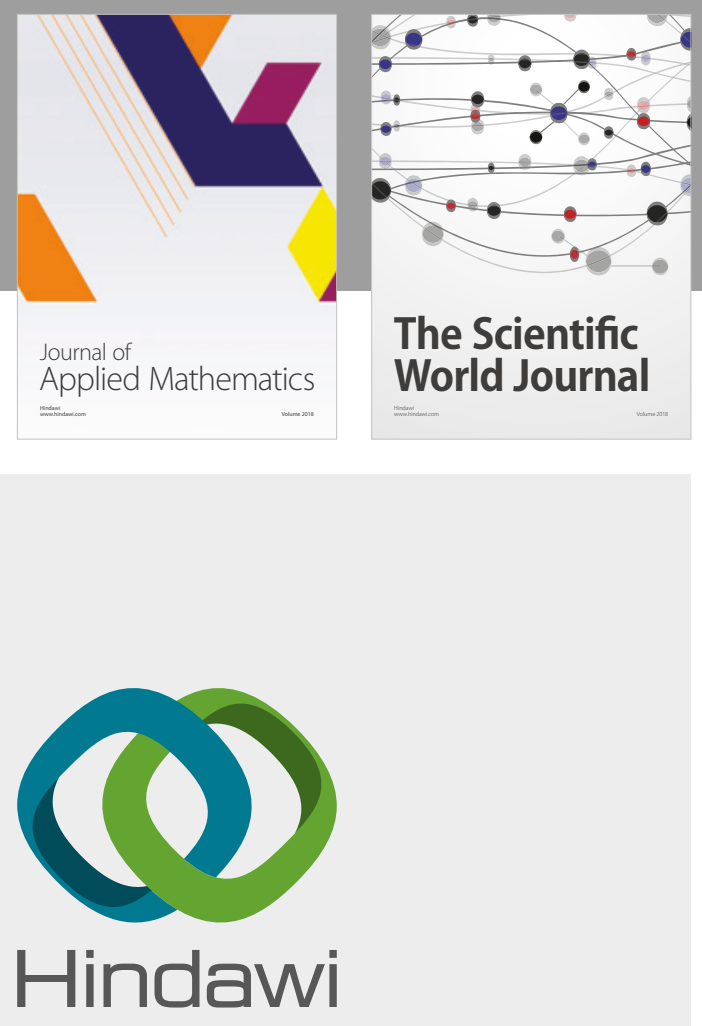

Submit your manuscripts at

www.hindawi.com

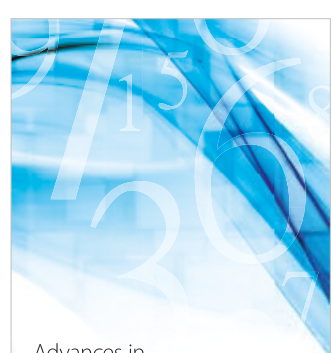

Advances in
Numerical Analysis
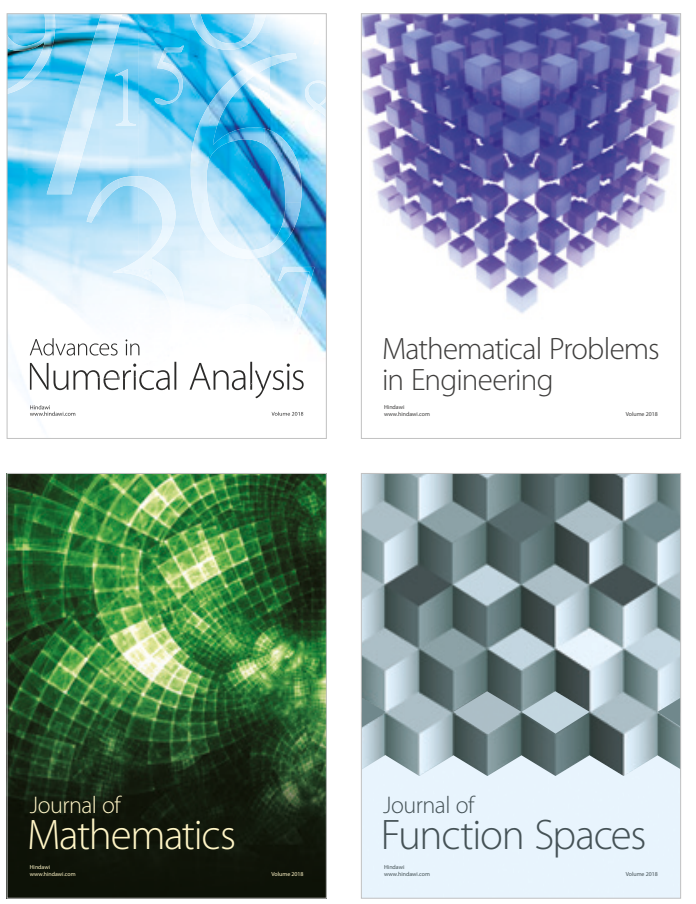

Mathematical Problems in Engineering

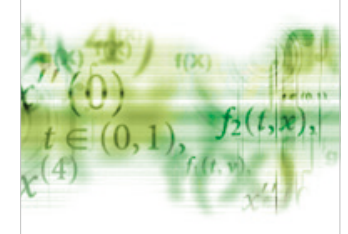

International Journal of

Differential Equations

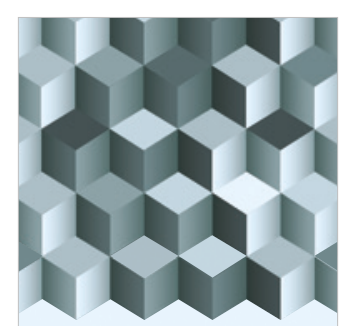

Journal of

Function Spaces

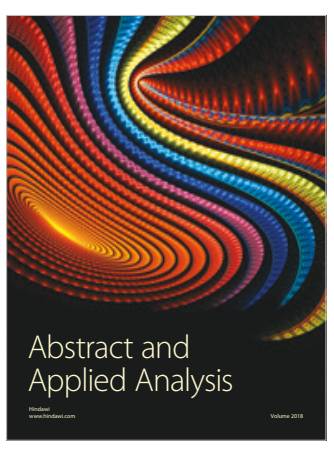

The Scientific

World Journal

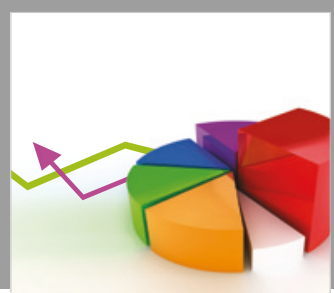

Journal of

Probability and Statistics
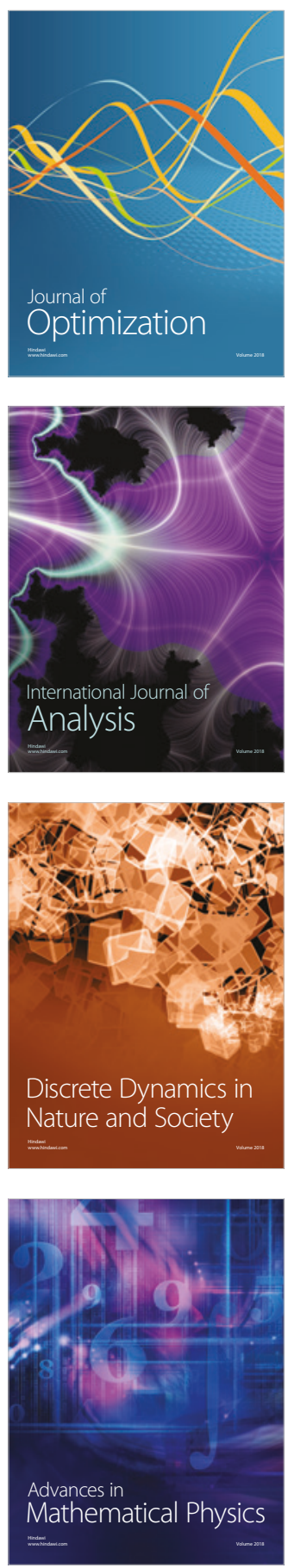